\section{Meldungen aus der SMS}

\section{SMS-Kongress in Tutzing}

\section{Unbedingt vormerken oder} gleich anmelden

Der 5. SMS-Kongress wird vom 6. - 8. Oktober 2017 in der Evangelischen Akademie in Tutzing am Starnberger See stattfinden.

Das Programm liegt bereits vor und ist anzufordern im Sekretariat der SMS, Franz-Josephstr. 38, D-80801 München, Tel (+49) 89 / 20083691 , Fax (+49) 89 / 80047681 , E-Mail:SMS@tcm.edu.

Das gesamte Programm ist auch im Internet zu finden unter www.tcm.edu und dort auch herunterzuladen.

\section{Neuer CTCA Newsletter}

Im März 2017 wurde ein neuer Newsletter vom Centrum für Therapiesicherheit in der Chinesischen Medizin (CTCA) veröffentlicht mit folgenden Themen: Liefereinschränkungen bei chinesischen Granulaten in Deutschland, Stellungnahme des CTCA zu einem grob verharmlosenden Artikel über die Aristolochia-Toxizität. Näheres unter www.ctca.de/index.php/de/aktuelles oder Anmeldung zum regelmäßigen Bezug des Newsletters unter info@ctca.de.

\section{Prüfung Diagnostik und Physiologie in Hamburg}

In Hamburg fand am 21. Januar 2017 die Prüfung Diagnostik und Physiologie der SMS statt, die folgende Teilnehmer erfolgreich ablegten: Dr. med Bozena Abel, Dr. med Maike Baumann, Dr. med Margret Fischer-Felten, Alina Goniksi, Anna Rehfeld und Dr. med Paul Wächtler.

Prüfung Akupunktur in München Am 11. Februar 2017 fand in München die Abschlussprüfung Akupunktur statt. Folgende Mitglieder legten sie erfolgreich ab: Rebecca Asadpour, Tamara Bähr, Julia Bykanova, Dr. med Claudia Feichtner, Karin Gärtner, Britte Kniefert, Dr.med. Daniela Lang-Rössle, Dr. med Cynthia Marisch, Dr. med Kerstin Moll, Gisela Peter, Sandra Spieß, Dr. med Daniela Waldhauser, Dr. med Maike Weißmann und Luisa Wikowski. (siehe Foto)

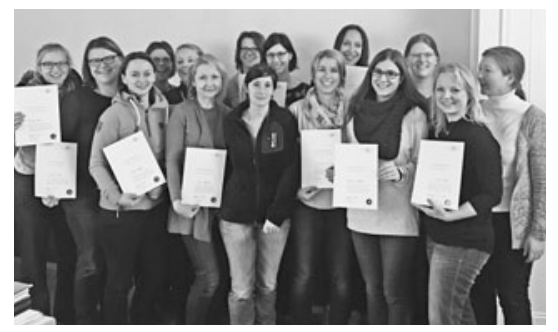

\section{Prüfung Diätetik-Zyklus in München}

Am 4. März 2017 fand in München die Diätetik-Prüfung der Offenen Schule der SMS statt, die folgende Teilnehmer erfolgreich ablegten: Nadine Göhlich, Manuela Hilz, Sabine Kauker, Erwin Kotter, Suza Ramsauer-Sewald, Daniela Ronke, Ulla Scharll, Antje Staab und Sophie Wattenbach.

\section{Abschlussprüfung Qigong- \\ Zyklus in Hamburg}

Am 1. April 2017 fand in Hamburg die Abschlussprüfung des Qigong-Zyklus der SMS statt. Folgende Teilnehmer absolvierten sie erfolgreich: Dr. med. Naomie Cayemitte-Rückner, Ulrike Christiansen, Annette Kettelhack, Dr. med Dunja Petersen und Dr. med. Michael Schöpper. (siehe Foto)

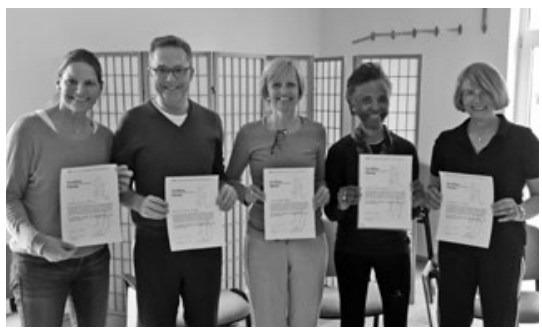

Ideenwettbewerb zur

Anwendung und Verbreitung

Chinesischer Arzneimittel -

Renommierte UCLA (University

College of Los Angeles) bietet

insgesamt 10.000US-\$ Preisgeld!

Am UCLA Center for East-West Medicine läuft eine Ausschreibung für schriftliche Beiträge zur Verbesserung der Anwendung, Sicherheit und Verbreitung Chinesischer Arzneimittel.

Wir wurden gebeten, dass die SMS ihren Mitgliedern und Interessierten diesen sinnvollen und attraktiven Wettbewerb bekannt macht.

Eine genaue Beschreibung und die Details zu diesem Wettbewerbes findet man unter:

www.

cewm.med.ucla.edu/news/herbcomp/

Die Deadline ist der 30. Juni 2017.

Nutzen Sie zum Beispiel als Student, als Arzt oder als Apotheker diese Gelegenheit, Ihre Kompetenz, Ihre Erfahrung und Ihre Ideen in Bezug auf die chinesische Arzneitherapie einem internationalen Gremium darzustellen! 